\title{
The Management and Outcome of Higher Order Multifetal Pregnancies: Obstetric, Neonatal and Follow-up Data
}

\author{
Mark H.Yudin', Elizabeth V.Asztalos ${ }^{2}$, Ann Jefferies ${ }^{3}$, and Jon F.R. Barrett ${ }^{4}$ \\ ${ }^{1-4}$ University of Toronto, Toronto, Ontario, Canada \\ 1,4 Department of Obstetrics and Gynecology \\ ${ }^{2,3}$ Departments of Newborn and Developmental Pediatrics and Neonatology \\ ${ }^{2,4}$ Women's College Campus of Sunnybrook and Women's College Health Sciences Center \\ ${ }^{3}$ Mount Sinai Hospital
}

\begin{abstract}
The objective of this study was to describe current obstetric, neonatal, and long-term neurodevelopmental outcomes of higher order multifetal gestations ( $\geq 3$ fetuses) in the 1990s. We also intended to identify a target gestational age at which neonatal and neurodevelopmental morbidities are low. Records from all multifetal pregnancies ( $\geq 3$ viable fetuses $\geq 20$ weeks gestation) delivered at the two perinatal centers in Toronto, Ontario, Canada during the study period (January 1, 1990-December 31, 1996) were reviewed. Data were collected on obstetric, neonatal, and long-term neurodevelopmental outcomes. Follow up data were gathered regarding the presence of a severe deficit in four categories (vision, hearing, cognition, and motor skills). Statistical analysis was performed to determine a gestational age at which a significant decrease in deficit occurred. During the study period 165 multifetal pregnancies were delivered. This resulted in 511 fetuses, of which 496 were live births. Of these 496 infants, 453 survived to discharge. Follow up data were obtained on 332 (73.3 per cent) infants. Infant survival increased with gestational age, and was approximately 90 per cent or greater at 26 weeks or more. Of all infants followed, the proportion of those without deficit increased with increasing gestational age, such that the per cent without deficit was 96.9 at 31 weeks or greater. Of all infants followed, 301 (90.7 per cent) had no deficit. Statistical analysis revealed a significant difference in long-term neurodevelopmental outcome between infants born before and after 28 weeks gestation. The incidence of a major deficit was 44.1 per cent for those born earlier than and 5.4 per cent for those born later than this gestational age $(p=0.001)$. In our cohort, survival figures were high. Even in lower gestational groupings, survival was high, but not without serious concerns about severe morbidity. This information is useful when counseling parents of higher order multifetal pregnancies.
\end{abstract}

While multiple births were formerly rare events, with the increased use of ovulation induction and in vitro fertilization techniques in recent years, their incidence has significantly increased. It is well known that an increase in the number of fetuses is associated with an increased incidence of maternal complications (Albrecht \& Tomich, 1996; Gonen et al., 1990; Itzkowic, 1979; Santema et al., 1995; Syrop and Varner, 1985), as well as increased perinatal morbidity and mortality (Gonen et al., 1990; Leonard et al., 1994; Lipitz et al., 1990; Loucopoulos \& Jewelewicz, 1982; Ron-El et al., 1981; Santema et al., 1995).

There is a growing body of literature on the management and outcome of higher order multifetal gestations. However, several of the existing studies have small sample sizes, and span many years. Obstetric and neonatal practices have changed considerably over time and may not reflect current standards of care. In addition, because of the increased use of reproductive technologies in recent years, obstetricians and neonatologists working in tertiary perinatal referral centers have been exposed to increasing numbers of multifetal gestations, providing them with invaluable experience. Finally, very few of the existing studies evaluate long-term follow-up for the infant. The objectives of this study were to describe current obstetric management, neonatal outcome, and long-term neurodevelopmental outcome of higher order multifetal gestations ( $\geq 3$ fetuses) in the 1990s, and to identify a gestational age at which neonatal and neurodevelopmental morbidities are low.

\section{Materials and Method}

This study was performed at the two sites of The University of Toronto Regional Perinatal Complex, the Women's College campus of the Sunnybrook and Women's College Health Sciences Center and Mount Sinai Hospital. These hospitals are the two tertiary perinatal referral centers in metropolitan Toronto, and serve Toronto itself, as well as the central eastern region of Ontario, Canada. Approximately 7000 births in total occur annually in the two centers, with more than 68000 occurring in the region.

All multifetal gestations delivered between January 1, 1990 and December 31, 1996 were included. Multifetal gestation was defined as a pregnancy with three or more viable fetuses at 20 weeks gestation or greater. Pregnancies

Address for correspondence: Elizabeth V. Asztalos, MD., Sunnybrook and Women's College Health Sciences Center, Women's College Campus, 76 Grenville Street, Room 466, Toronto, Ontario M5S 1B2, Canada. 
in which delivery or death of one or more fetus occurred before 20 weeks gestation were excluded. Pregnancies were identified through delivery suite and neonatal intensive care unit (NICU) logbooks, and through obstetrical and neonatal databases. Once eligible pregnancies were identified, all obstetric and neonatal data were obtained by retrospective chart review. The data included information relating to antepartum, intrapartum, postpartum and neonatal management and complications. For the follow-up data, information was gathered through a combination of retrospective chart review from the follow-up clinics and direct contact with families. The data included information relating to four major deficits: vision, hearing, cognition and motor skills. Children born at Women's College Hospital were followed if at least one child from the gestation had a birth weight of less than 1500 grams. Children born at Mount Sinai Hospital were followed if at least one child from the gestation had a birth weight of less than 1250 grams. To ensure consistency, only two of the authors (MHY, EVA) collected all data. Once collected, the data were organized using Microsoft Excel software (Microsoft Corp., Redmond, WA). Our research protocol was approved by the research ethics boards of each institution.

For the obstetric data, clinical diagnoses were made by attending staff obstetricians according to standard definitions. For the neonatal data, clinical diagnoses were made by attending staff neonatologists according to standard definitions (Bell et al., 1978; Faranoff \& Martin, 1997; The Committee for the Classification of Retinopathy of Prematurity, 1984; Volpe, 1995) and are described in Table 1. For assigning birth order, $\mathrm{A}$ was considered the first infant delivered, followed by B, C, etc. Small for gestational age (SGA) was diagnosed when birth weight was less than two standard deviations from the mean for gestational age (Usher \& McLean, 1969). Vital statistics were calculated for the perinatal data. The stillbirth rate was defined as the number of stillbirths per 1000 births (live and stillbirths). The early neonatal mortality rate was defined as the number of deaths of live-born infants weighing 500 grams or more during the first seven days of life per 1000 liveborn infants. The late neonatal mortality rate was defined as the number of deaths of live-born infants weighing 500 grams or more after seven days and before 28 days of life per 1000 live-born infants. The perinatal mortality rate was defined as the number of stillbirths and early neonatal deaths per 1000 total births.

For the follow-up data, a major visual deficit was defined as vision $<20 / 200$ in one or both eyes. A major hearing deficit was defined as the need for amplification based on a formal hearing assessment. A major cognitive deficit was based on a score of less than two standard deviations from the mean on a neuro-cognitive assessment. All children followed at Women's College Hospital underwent the Bayley Developmental Assessment at 18 months corrected age. Version I was used until 1995 and Version II was used from then on (Bayley, 1969; 1993). All children followed at Mount Sinai Hospital underwent the Diagnostic Inventory for Screening Children (DISC) at 21 months corrected age (Parker et al., 1990). For those children who had not been followed or undergone such testing, cognitive assessment was based on their report cards from school. A major motor deficit was defined as presence of abnormal tone interfering with ambulation consistent with cerebral palsy.

Descriptive statistics (means, standard deviations, and proportions) were used to describe the study population. The primary outcome measure was the presence of a serious long-term neurodevelopmental deficit in at least one of four categories (vision, hearing, cognition, and motor skills). The proportion of infants with a deficit at each level of gestational age (27 weeks vs. 28 weeks, 28 weeks vs. 29 weeks, etc.) was compared using a chi-square statistic to determine at what gestational age a significant

\section{Table 1}

Definitions of Neonatal Complications

Respiratory Distress Syndrome (RDS)

Transient Tachypnea of the Newborn (TTN)

Chronic Lung Disease (CLD)

Patent Ductus Arteriosus (PDA)

Intraventricular Hemorrhage (IVH)

Necrotizing Enterocolitis

Retinopathy of Prematurity (ROP)
Respiratory distress requiring oxygen therapy is present beyond the first 24 hours of life, with typical radiographic signs (Faranoff and Martin, 1997)

Respiratory distress requiring oxygen therapy is present for less than the first 24 hours of life, with no radiographic signs of RDS (Faranoff and Martin, 1997)

Respiratory sequelae requiring oxygen therapy is present at 36 weeks corrected gestational age (Faranoff and Martin, 1997)

Confirmed by echocardiographic visualization of a patent ductus

Confirmed by head ultrasound as follows: grade 1 involving germinal matrix hemorrhage occupying less than 10 per cent of ventricular area, grade 2 involving IVH occupying 10-50 per cent ventricular area, grade 3 involving IVH occupying more than 50 per cent of ventricular area, and grade 4 involving periventricular hemorrhagic infarction of adjacent white matter (Volpe, 1995).

Clinical gastrointestinal disturbances associated with pneumatosis intestinalis on abdominal radiography (Bell et al., 1978)

Defined on the basis of the criteria of the Committee for the Classification of Retinopathy of Prematurity (The Committee for the Classification of Retinopathy of Prematurity, 1984). 
decrease in deficit occurred. Data were combined for the weeks 24-26 and 36-37 due to small numbers. The level of statistical significance was set at 0.05 . Because a significant difference was found in long-term neurodevelopmental outcome between infants born before and after 28 weeks gestation, the proportion of neonatal outcomes before and after 28 weeks gestation were compared using a chi-square statistic to examine the morbidity of the less than 28 week gestation neonate. Infants born vaginally were compared to infants born by cesarean section with respect to neurodevelopmental deficit and neonatal outcome using a chi-square statistic. First vaginally born infants were compared to subsequent vaginally born infants with respect to neurodevelopmental deficit and neonatal outcome using a Fisher's Exact test.

\section{Results}

From January 1, 1990 to December 31, 1996, there were 165 higher order multifetal pregnancies delivered, resulting in 511 fetuses. There were 150 sets of triplets, 14 sets of quadruplets, and one set of quintuplets. One hundred sixty three charts were reviewed, as medical records could not locate two charts. Follow-up data were available on 332 children (73.3 per cent of those surviving to discharge from hospital). Most of these children were followed in the follow-up clinics. Attempts were made to locate children not followed, using phone numbers and family doctors listed in the hospital charts, searching telephone books, and contacting national multifetal gestation registries. The most common reason for an inability to locate children was that the family had moved from the last recorded address with no forwarding address.

The mean maternal age was 31.0 years (range 16-41). The majority of pregnancies (76.4\%) were conceived with some form of assisted reproduction, with the remainder conceived spontaneously. The majority of the spontaneously conceived pregnancies were triplet pregnancies. All but one of the quadruplet pregnancies and the one quintuplet pregnancy were conceived with assisted reproduction.

\section{Antenatal Management and Maternal Complications}

With respect to antenatal management, steroids were used in $93.4 \%$ of pregnancies. A large number of women were admitted to hospital, with $84.0 \%$ having at least one admission prior to delivery. The most frequent reason was elective admission for bedrest $(26.3 \%)$, followed by threatened preterm labour (20.4\%). Other common reasons for admission to hospital included cervical dilatation, effacement or funneling $(17.5 \%)$, preeclampsia or pregnancy-induced hypertension $(\mathrm{PIH})(9.5 \%)$, and poor fetal growth $(5.1 \%)$. Most women requiring hospital admission were only admitted once, although 27 women were admitted twice and seven were admitted three times. The vast majority of women $(97.9 \%)$ were placed on bedrest, either at home or in hospital.

With respect to maternal morbidity and complications, preterm labour occurred in $67.5 \%$ and premature rupture of membranes (PROM) in $31.3 \%$ of cases. Preeclampsia or $\mathrm{PIH}$ was noted in $16.6 \%$ of pregnancies, and gestational diabetes mellitus in $7.4 \%$. Anemia (defined as hemoglobin of $90 \mathrm{~g} / \mathrm{dl}$ or less) occurred in $8.0 \%$ of cases, incompetent cervix requiring a suture in $4.3 \%$, and hyperemesis gravidarum in $3.7 \%$.

\section{Labour, Delivery and Postpartum}

The average gestational age at delivery was 31.4 weeks (range 20.1-37.4). Most pregnancies (87.7\%) were delivered by Cesarean section. The most common reason for delivery was preterm labour (32.5 per cent) or a combination of preterm labour and PROM (14.7\%). Approximately $15 \%$ of pregnancies were terminated with an elective Cesarean section. The gestational age for these pregnancies ranged from 33.0 to 37.4 weeks. The most common reason for elective Cesarean section was maternal discomfort. There were 20 pregnancies in which at least one infant was delivered vaginally. These were all triplet pregnancies. In three pregnancies, triplet A was delivered vaginally and $\mathrm{B}$ and $\mathrm{C}$ were delivered by Cesarean section. In the other 17 , all three triplets were delivered vaginally. The average gestational age at delivery for these pregnancies was 25.7 weeks (range 20.1-32.1). During labour and delivery, there were five cases of abruptio placenta but no cases of cord prolapse.

The most commonly encountered postpartum complications were postpartum fever of $38.5^{\circ} \mathrm{C}$ or more in $11.7 \%$ and postpartum hemorrhage and blood transfusion in $4.3 \%$ of pregnancies. There were five cases of culture-positive maternal sepsis, six cases of culture-positive maternal urinary tract infection, seven cases of endometritis and five cases of wound infection. There were three cases of wound hematoma, two cases of pelvic hematoma, and one case of pulmonary embolus.

\section{Neonatal Outcome}

The 165 pregnancies resulted in a total of 511 fetuses. There were 496 live births and 15 stillbirths. Four hundred and fifty three infants survived to discharge from hospital, representing $88.6 \%$ of all fetuses and $91.3 \%$ of all live births. The stillbirth rate was 31.4 per 1000 births, the early neonatal mortality rate was 44.5 and the late neonatal mortality rate was 2.0 per 1000 live births, and the perinatal mortality rate was 107.8 per 1000 total births. Table 2 describes neonatal survival based on gestational age. The average number of days spent in hospital was 31.9 (range 1-291). The male/female ratio was 1.04 . Table 3 lists the mean birth weights by gestational age. As expected, the mean birth weight rose with gestational age. The incidence of small for gestational age (SGA) infants was 25.3 per cent. The average Apgar scores were six at one minute and eight at five minutes. There were 18 infants with congenital anomalies.

\section{Neonatal Complications}

The incidence of respiratory distress syndrome (RDS) was $39.1 \%$, transient tachypnea of the newborn (TTN) 11.3\%, and chronic lung disease (CLD) 6.8\%. Surfactant was given in $36.6 \%$ of cases, and some form of ventilatory support was required in $55.1 \%$ of cases. The incidence of patent ductus arteriosus (PDA) was $22.1 \%$, with $67.3 \%$ of the cases requiring indomethacin and $2.9 \%$ requiring surgical ligation. The incidence of congenital heart disease was 
Table 2

Gestational Age and Neonatal Survival

\begin{tabular}{ccc}
\hline $\begin{array}{c}\text { Gestational Age } \\
\text { (weeks) }\end{array}$ & $\begin{array}{c}\text { Total Number } \\
\text { of Fetuses }\end{array}$ & $\begin{array}{c}\text { Number (\%) } \\
\text { Survival }\end{array}$ \\
\hline$<24$ & 24 & $1(0.4)$ \\
24 & 6 & $2(33.3)$ \\
25 & 9 & $6(66.7)$ \\
26 & 9 & $8(88.9)$ \\
27 & 39 & $34(87.2)$ \\
28 & 34 & $32(91.2)$ \\
29 & 43 & $37(86.1)$ \\
30 & 50 & $48(96.0)$ \\
$\geq 31$ & 296 & $287(96.9)$ \\
\hline
\end{tabular}

Table 3

Gestational Age and Birth Weight

\begin{tabular}{cc}
\hline $\begin{array}{c}\text { Gestational Age } \\
\text { (weeks) }\end{array}$ & $\begin{array}{c}\text { Average Birth Weight } \\
(\mathrm{g})\end{array}$ \\
\hline 24 & 485.00 \\
25 & 722.50 \\
26 & 738.17 \\
27 & 864.00 \\
28 & 1027.25 \\
29 & 1148.74 \\
30 & 1278.18 \\
31 & 1398.87 \\
32 & 1661.28 \\
33 & 1621.16 \\
34 & 1778.06 \\
35 & 1916.52 \\
36 & 2008.26 \\
37 & 1940.00 \\
\hline
\end{tabular}

2.8\%. The incidence of sepsis was $15.7 \%$, with $91.9 \%$ of the cases being bacterial, $5.4 \%$ viral and $2.7 \%$ fungal. The incidence of necrotizing enterocolitis was $2.6 \%$, retinopathy of prematurity (ROP) $13.0 \%$, and intraventricular hemorrhage (IVH) 8.1\%.

Table 4 compares the incidence of major neonatal complications for infants born vaginally and by Cesarean section. Although the number of infants in the vaginally born group was very small, there was a significantly higher incidence of several complications (RDS, need for surfactant, need for ventilatory support, PDA, IVH) in this group. There was also a significantly higher chance of survival to discharge from hospital in the Cesarean section group. Table 5 compares the incidence of major neonatal complications by birth order in the vaginally born group of infants. For most categories, we could not demonstrate a significant difference between the first and subsequently born infants, although there was a significantly lower incidence of RDS and surfactant use in the first born infants.

\section{Neurodevelopmental Outcome}

Four hundred and fifty three infants survived to discharge from hospital, and follow-up data were available on 332 (73.3 per cent) of these. Length of time of follow-up ranged from nine months to 8.5 years. In total, 301 $(90.7 \%)$ of the 332 children followed are free from major deficits at follow-up. The incidence of a major visual deficit was $2.1 \%$, a major hearing deficit $0.6 \%$ and a major cognitive deficit $3.3 \%$. The incidence of cerebral palsy was $7.8 \%$, $0.9 \%$ of children required the use of a wheelchair and $0.6 \%$ required the use of some other motor aid. The median age at walking was approximately 12 months of age, and $90.0 \%$ of children were walking by 17 months. Table 6 describes neurodevelopmental outcome as a function of gestational age at birth. For infants born at 28 weeks gestation or greater, the rate of severe morbidity was $12.5 \%$, and for those born at 31 weeks or greater, the rate was $9.3 \%$. Chi-square analysis revealed that there was a significant dif-

Table 4

Comparison of Neonatal Complications for Infants Born Vaginally and by Cesarean Section

\begin{tabular}{|c|c|c|c|}
\hline Complication & Vaginal Delivery (\%) & Cesarean Section (\%) & $p$ Value \\
\hline \multicolumn{4}{|l|}{ Respiratory } \\
\hline RDS & 74.2 & 36.2 & 0.001 \\
\hline TTN & 9.7 & 11.1 & 0.812 \\
\hline Surfactant & 74.2 & 33.4 & 0.001 \\
\hline Ventilatory Support & 90.3 & 51.8 & 0.001 \\
\hline \multicolumn{4}{|l|}{ Cardiovascular } \\
\hline PDA & 46.7 & 19.8 & 0.001 \\
\hline \multicolumn{4}{|l|}{ Central Nervous System } \\
\hline IVH & 23.3 & 6.7 & 0.001 \\
\hline \multicolumn{4}{|l|}{ Gastrointestinal } \\
\hline Necrotizing Enterocolitis & 6.7 & 2.3 & 0.145 \\
\hline \multicolumn{4}{|l|}{ Eye } \\
\hline ROP & 16.7 & 12.4 & 0.502 \\
\hline \multicolumn{4}{|l|}{ Sepsis } \\
\hline Sepsis & 16.7 & 13.8 & 0.664 \\
\hline Survival to Discharge & 38.3 & 95.7 & 0.001 \\
\hline Neurodevelopmental Deficit & 20.0 & 8.2 & 0.073 \\
\hline
\end{tabular}


Table 5

Comparison of Neonatal Complications by Birth Order for Infants Born Vaginally

\begin{tabular}{|c|c|c|c|}
\hline Complication & Infant A (\%) & Infants B and C (\%) & $p$ Value \\
\hline SGA & 31.3 & 40.0 & 0.549 \\
\hline \multicolumn{4}{|l|}{ Respiratory } \\
\hline RDS & 45.5 & 90.0 & 0.007 \\
\hline TTN & 18.2 & 5.0 & 0.235 \\
\hline Surfactant & 45.5 & 90.0 & 0.007 \\
\hline Ventilatory Support & 81.8 & 95.0 & 0.235 \\
\hline \multicolumn{4}{|l|}{ Cardiovascular } \\
\hline PDA & 54.6 & 42.1 & 0.510 \\
\hline \multicolumn{4}{|l|}{ Central Nervous System } \\
\hline IVH & 18.2 & 26.3 & 0.612 \\
\hline \multicolumn{4}{|l|}{ Gastrointestinal } \\
\hline Necrotizing Enterocolitis & 0.0 & 10.5 & 0.265 \\
\hline \multicolumn{4}{|l|}{ Eye } \\
\hline ROP & 27.3 & 10.5 & 0.236 \\
\hline \multicolumn{4}{|l|}{ Sepsis } \\
\hline Sepsis & 9.1 & 21.1 & 0.397 \\
\hline Survival to Discharge & 50.0 & 32.5 & 0.189 \\
\hline Neurodevelopmental Deficit & 22.2 & 18.2 & 0.822 \\
\hline
\end{tabular}

Table 6

Neurodevelopmental Summary

\begin{tabular}{|c|c|c|c|c|c|}
\hline $\begin{array}{l}\text { Gestational Age } \\
\text { (weeks) }\end{array}$ & Total Born & Survival (\%) & Followed & Deficit & No Deficit (\%) \\
\hline$\leq 23$ & 24 & $1(0.4)$ & 0 & 0 & $0(0)$ \\
\hline 24 & 6 & $2(33.3)$ & 1 & 0 & $1(100.0)$ \\
\hline 25 & 9 & $6(66.7)$ & 4 & 3 & $1(25.0)$ \\
\hline 26 & 9 & $8(88.9)$ & 6 & 3 & $3(50.0)$ \\
\hline 27 & 39 & $34(87.2)$ & 23 & 9 & $14(60.9)$ \\
\hline 28 & 34 & $32(91.2)$ & 32 & 4 & $28(87.5)$ \\
\hline 29 & 43 & $37(86.1)$ & 34 & 3 & 31 (91.2) \\
\hline 30 & 50 & $48(96.0)$ & 40 & 3 & $37(92.5)$ \\
\hline$\geq 31$ & 296 & $287(96.9)$ & 192 & 6 & $186(96.9)$ \\
\hline
\end{tabular}

ference in long-term neurodevelopmental outcome between infants born before and after 28 weeks gestation. The incidence of a major deficit was $44.1 \%$ for those born earlier than and $5.4 \%$ for those born later than this gestational age $(p=0.001)$. Table 7 compares the incidence of major neonatal complications for infants born before and after 28 weeks gestation.

\section{Discussion}

The incidence of multifetal gestation has significantly increased in recent years. Because of this, there is a great need for current data on management and outcome. Much of the existing literature comes from studies with small sample sizes (Byrne et al., 1993; Lipitz et al., 1990; Ron-El et al., 1981; Santema et al., 1995; Sassoon et al., 1990) spanning many years (Byrne et al., 1993; Itzkowic, 1979; Lipitz et al., 1989, 1990; Newman et al., 1989; Parker et al., 1990; Ron-El et al., 1981; Santema et al., 1995;
Sassoon et al., 1990). Practices have changed considerably and there have been many advances in obstetric and neonatal care over this period, and these studies may therefore not reflect current standards of care or outcomes. Finally, very few studies have evaluated long-term neurodevelopmental outcome (Collins \& Bleyl, 1990; Leonard et al., 1994; Lipitz et al., 1990; Sassoon et al., 1990; Syrop \& Varner, 1985; Wildschut et al., 1995). For all of these reasons, we felt this topic required further investigation. The purpose of this study was to describe current obstetric, neonatal, and long-term neurodevelopmental outcomes of higher order multifetal gestations in the 1990s, and to identify a gestational age at which neonatal and neurodevelopmental morbidities are low.

An increase in the number of fetuses is associated with an increased incidence of maternal complications (Albrecht \& Tomich, 1996; Gonen et al., 1990; Itzkowic, 1979; Santema et al., 1995; Syrop \& Varner, 1985). The aim of obstetric management is to minimize this morbidity. In 
Table 7

Comparison of Neonatal Complications for Infants Born Before and After 28 Weeks Gestation

\begin{tabular}{|c|c|c|c|}
\hline Complication & $<28$ weeks $(\%)$ & $\geq 28$ weeks $(\%)$ & $p$ value \\
\hline$\overline{S G A}$ & 27.9 & 23.1 & 0.363 \\
\hline \multicolumn{4}{|l|}{ Respiratory } \\
\hline RDS & 90.3 & 31.3 & 0.001 \\
\hline TTN & 4.8 & 12.2 & 0.086 \\
\hline Surfactant & 83.9 & 29.3 & 0.001 \\
\hline Ventilatory Support & 100.0 & 48.2 & 0.001 \\
\hline \multicolumn{4}{|l|}{ Cardiovascular } \\
\hline PDA & 61.3 & 16.2 & 0.001 \\
\hline \multicolumn{4}{|l|}{ Central Nervous System } \\
\hline IVH & 30.7 & 4.7 & 0.001 \\
\hline \multicolumn{4}{|l|}{ Gastrointestinal } \\
\hline Necrotizing Enterocolitis & 8.1 & 1.7 & 0.003 \\
\hline \multicolumn{4}{|l|}{ Eye } \\
\hline ROP & 46.8 & 7.8 & 0.001 \\
\hline \multicolumn{4}{|l|}{ Sepsis } \\
\hline Sepsis & 46.8 & 9.6 & 0.001 \\
\hline Survival to Discharge & 58.6 & 95.3 & 0.001 \\
\hline Neurodevelopmental Deficit & 44.1 & 5.4 & 0.001 \\
\hline
\end{tabular}

earlier studies (Byrne et al., 1993; Gonen et al., 1990; Itzkowic, 1979; Sassoon et al., 1990), use of prophylactic measures such as elective bed rest and hospital admission was widespread. In later studies (Albrecht \& Tomich, 1996; Santema et al., 1995), management was often geared toward the treatment of complications only when they occurred, and it was emphasized that prophylactic measures probably have little benefit in prolonging multifetal gestation (Byrne et al., 1993; Itzkowic, 1979; Newman et al, 1989). Despite this, $84.0 \%$ of women in our series were admitted to hospital at least once, and the most common reason was an elective admission for bed rest. The next most common reason for admission to hospital was threatened preterm labour. Indeed, preterm labour is the most common pregnancy complication identified in virtually every study, with rates usually greater than $90.0 \%$ (Collins \& Bleyl, 1990; Gonen et al, 1990; Santema et al., 1995). In total, preterm labour occurred in $67.5 \%$ of pregnancies in our series, and was also the most common reason for delivery. The incidence of other commonly reported complications including PROM, preeclampsia, anemia, and postpartum hemorrhage compares favourably with the literature, and was less than that reported in many previous studies (Albrecht \& Tomich, 1996; Byrne et al., 1993; Collins \& Bleyl, 1990; Ron-El et al., 1981; Santema et al., 1995). This finding may be explained by the fact that earlier studies did not have as large a sample size as the present study.

The optimal mode of delivery of higher order multifetal gestations is still controversial. In many early studies, the vaginal route was chosen as the preferred mode of delivery (Itzkowic, 1979; Ron-El et al., 1981). In later series, however, there has been a dramatic increase in cesarean section rates (Gonen et al., 1990; Lipitz et al., 1989, 1990; Newman et al., 1989; Sassoon et al., 1990). In our series, cesarean section was the mode of delivery in $87.7 \%$ of cases.
We found a significantly higher incidence of RDS, surfactant use, the need for ventilatory support, PDA and IVH in those infants born vaginally compared to those born by cesarean section. As well, survival to discharge from hospital was greater in those born by cesarean section. It is important to note, however, that the number of vaginally born infants in our series was very small, and these results should be interpreted with caution. For those infants born vaginally, we were unable to demonstrate a significant difference in neonatal complications between the first-born and subsequently born infants, except for a higher incidence of RDS and surfactant use in the subsequently born infants. Although some studies have demonstrated increased morbidity and mortality for the second and third vaginally born triplet (Itzkowic, 1979; Lipitz et al., 1989; Ron-El et al., 1981), the answer to this question is still unclear.

In our series, the stillbirth, early and late neonatal mortality, and perinatal mortality rates compare favourably with the literature. The proportion of infants surviving to discharge from hospital was very high, but there are still serious concerns about morbidity. Generally, as the number of fetuses increases, the duration of gestation and birthweight of each fetus decreases (Collins and Bleyl, 1990; Lipitz et al., 1990; Sassoon et al., 1990; Syrop \& Varner, 1985). Birthweight has been shown to be one of the most important factors affecting perinatal mortality and the most significant determinant of infant and childhood morbidity (Luke \& Keith, 1992; Akerman et al., 1995). Compared with infants of normal weight, low birthweight infants (LBW, < 2500 grams) are nearly 40 times as likely to die in the neonatal period (McCormick et al., 1985; Shapiro et al., 1980). In our series, the incidence of SGA infants was $25.3 \%$. Although neonatal morbidity in this series compared favourably to that reported elsewhere in the literature (Albrecht \& Tomich, 1996; Gonen et al., 1990; Kaufman et al., 1998), it was still prevalent enough to be of concern. 
Follow-up data were available on 332 of 453 infants (73.3\%). Length of time of follow-up ranged from nine months to 8.5 years. Among survivors of the neonatal period, LBW infants are three times as likely to exhibit neurodevelopmental handicaps, and the risk increases with declining birthweight (Taylor, 1984). In addition, LBW children experience more chronic illnesses throughout childhood (Overpeck et al., 1989). Despite this discouraging prognosis, the results of most studies involving higher order multifetal pregnancies have been quite favorable with respect to long-term outcome. Most recent studies have found that $70-75 \%$ of children are free from major neurologic or developmental handicaps at an age of least two years (Gonen et al., 1990; Lipitz et al., 1990; Luke \& Keith, 1992). Our results revealed that the proportion of infants without long-term deficits increased with gestational age at birth. For infants born at 28 weeks or gestation or greater, the rate of severe long-term morbidity was 12.5 per cent, and for those born at 31 weeks or greater, the rate was only 9.3 per cent. Most of the morbidity was associated with cerebral palsy and deficits in motor skills.

Statistical analysis demonstrated that there was a significant difference in long-term neurodevelopmental outcome between infants born before and after 28 weeks gestation ( 44.1 vs. $5.4 \%, p=0.001$ ). The large number of infants followed over time lends support to this statement. This gestational age may be used as a possible target with the knowledge that neonatal and neurodevelopmental morbidities are relatively low once this point is reached. This information is useful not only to clinicians, but also to parents of higher order multifetal pregnancies. To our knowledge, no other studies reported so far have attempted to identify a gestational age threshold for morbidity in this manner.

Because this was not a randomized control trial, it is therefore open to selection bias. Women with higher order multifetal pregnancies are less likely to deliver at community hospitals and more likely to be cared for at tertiary perinatal referral centers. It is important to note, however, that the perinatal system in Ontario, Canada is structured such that women identified as having pregnancies at risk are referred to a designated tertiary perinatal referral center in their region and are not usually managed or delivered at a community hospital. Infants of mothers treated or delivered at tertiary centers may have a better outcome because of the availability of a neonatal intensive care unit and optimal resuscitation conditions. Therefore, these data may not be generalizable to all infants. As well, various treatments (such as antenatal steroids, tocolytics, and antibiotics) and certain modes of delivery (such as cesarean section) may be preferentially used in certain cases (such as higher order multifetal gestations or more premature cases), and these cases may be associated with a worse outcome. A final limitation of the study is that follow-up data were available in only $73.3 \%$ of cases. This was largely due to difficulties in locating families whose children were not followed in the follow-up clinics despite repeated attempts and various methods. It is important to note that because of established criteria for neonatal follow-up, infants of lower weights and gestational ages were more likely to be followed, and these were the infants at highest risk for mor- bidity. The greatest proportion of infants lost to follow-up had weights of greater than 1500 grams, and the trend in this group was a very low incidence of neonatal and neurodevelopmental morbidity.

The strengths of this study lie in its numbers and its follow-up data. No other studies have had as large a sample size, and this increases the power of the results. Also, no other studies have had as large a cohort to report on longterm neurodevelopmental outcome, or attempted to identify a gestational age threshold after which morbidity is low.

Despite the limitations of the study, the results are encouraging and can be used to assist in counseling parents of higher order multifetal gestations. Women with these pregnancies should be cared for and delivered at tertiary perinatal referral centers for optimal outcomes. Analysis of data in this study suggests that clinicians and parents can identify 28 weeks as a target gestational age threshold to aim for in order to reduce neonatal and neurodevelopmental complications.

\section{Acknowledgments}

The authors would like to acknowledge the help of Dr. Arne Ohlsson for his assistance with preparation of the manuscript, and Ms. Terry Leeke and Ms. Terri Myhr for their assistance with data set-up and analysis.

\section{$\overline{\text { References }}$}

Akerman, B.A., Hovmoller, M., Radestad, A., \& Thomassen, P.A. (1995). Physical and mental development in 4-6-year-old triplets. Acta Paediatrica, 84, 661-666.

Albrecht, J.L., \& Tomich, P.G. (1996). The maternal and neonatal outcome of triplet gestations. American Journal of Obstetrics and Gynecology, 174, 1551-1556.

Bayley, N. (1969). Bayley scales of infant development. New York: The Psychological Corporation.

Bayley, N. (1993). Bayley scales of infant development. 2nd ed. San Antonio: The Psychological Corporation, Harcourt Brace and Company.

Bell, M.J., Ternberg, J.L., Feigin, R.D., Keating, J.P., Marshall, R., Barton, L., \& Brotherton, T. (1978). Neonatal necrotizing enterocolitis: therapeutic decisions based upon clinical staging. Annals of Surgery, 187, 1-7.

Byrne, B.M., Rasmussen, M.J., \& Stronge, J.M. (1993). A review of triplet pregnancy. Irish Medical Journal, 86, 55-57.

Collins, M.S., \& Bleyl, J.A. (1990). Seventy-one quadruplet pregnancies: management and outcome. American Journal of Obstetrics and Gynecology, 162, 1384-1392.

Faranoff, A.A., \& Martin, R.J. (1997). Neonatal-perinatal medicine: diseases of the fetus and infant. 6th ed. St. Louis, Missouri: Mosby-Year Book.

Gonen, R., Heyman, E., Asztalos, E.V., Ohlsson, A., Pitson, L.C., Shennan, A.T., \& Milligan, J.E. (1990). The outcome of triplet, quadruplet, and quintuplet pregnancies managed in a perinatal unit: obstetric, neonatal, and follow-up data. American Journal of Obstetrics and Gynecology, 162, 454-459.

Itzkowic, D. (1979). A survey of 59 triplet pregnancies. British Journal of Obstetrics and Gynaecology, 86, 23-28.

Kaufman, G.E., Malone, F.D., Harvey-Wildes, K.B., Chelmow, D., Penzias, A.S., \& D'Alton, M.E. (1998). Neonatal morbid- 
ity and mortality associated with triplet pregnancy. Obstetrics and Gynecology, 91, 342-348.

Leonard, C.H., Piecuch, R.E., Ballard, R.A., \& Cooper, B.A.B. (1994). Outcome of very low birth weight infants: multiple gestation versus singletons. Pediatrics, 93, 611-615.

Lipitz, S., Frenkel, Y., Watts, C., Ben-Rafael, Z., Barkai, G., \& Reichman, B. (1990). High-order multifetal gestation- management and outcome. Obstetrics and Gynecology, 76, 215-218.

Lipitz, S., Reichman, B., Paret, G., Modan, M., Shalev, J., Serr, D.M., Mashiach, S., \& Frenkel, Y. (1989). The improving outcome of triplet pregnancies. American Journal of Obstetrics and Gynecology, 161, 1279-1284.

Loucopoulos, A., \& Jewelewicz, R. (1982). Management of multifetal pregnancies: sixteen years' experience at the Sloane Hospital for Women. American Journal of Obstetrics and Gynecology, 143, 902-905.

Luke, B., \& Keith, L.G. (1992). The contribution of singletons, twins and triplets to low birth weight, infant mortality and handicap in the United States. Journal of Reproductive Medicine, 37, 661-666.

McCormick, M.C. (1985). The contribution of low birth weight to infant mortality and childhood morbidity. New England Journal of Medicine, 312, 82-90.

Newman, R.B., Hamer, C., \& Miller, M.C. (1989). Outpatient triplet management: a contemporary review. American Journal of Obstetrics and Gynecology, 161, 547-555.

Overpeck, M.D., Moss, A.J., Hoffman, H.J., \& Hendershot, G.E. (1989). A comparison of the childhood health status of normal birthweight and low birthweight infants. Public Health Reports, 104, 58-70.

Parker, K.C.H., Mainland, M.K., \& Amdur, J.R. (1990). DISC Preschool Screen, Version 3.0. St. Clements: The Psychological Corporation.
Ron-El, R., Caspi, E., Schreyer, P., Weinraub, Z., Arieli, S., \& Goldberg, M. (1981). Triplet and quadruplet pregnancies and management. Obstetrics and Gynecology, 57, 458-463.

Santema, J.G., Bourdrez, P., \& Wallenburg, H.C.S. (1995). Maternal and perinatal complications in triplet compared with twin pregnancy. European Journal of Obstetrics, Gynecology and Reproductive Biology, 60, 143-147.

Sassoon, D.A., Castro, L.C., Davis, J.L., \& Hobel, C.J. (1990). Perinatal outcome in triplet versus twin gestations. Obstetrics and Gynecology, 75, 817-820.

Shapiro, S., McCormick, M.C., Starfield, B.H., Krischer, J.P., \& Bross, D. (1980). Relevance of correlates of infant deaths for significant morbidity at one year of age. American Journal of Obstetrics and Gynecology, 136, 363-373.

Syrop, C.H., \& Varner, M.W. (1985). Triplet gestation: maternal and neonatal implications. Acta Geneticae Medicae et Gemellologiae, 34, 81-88.

Taylor, D.J. (1984). Low birthweight and neurodevelopmental handicap. Clinical Obstetrics and Gynecology, 11, 525-542.

The Committee for the Classification of Retinopathy of Prematurity. (1984). An international classification for retinopathy of prematurity. Archives of Ophthalmology, 102, 1130-1134.

Usher, R., \& McLean, F. (1969). Intrauterine growth of live-born caucasian infants at sea level: standards obtained from measurements on 7 dimensions of infants born between 25 and 44 weeks of gestation. Journal of Pediatrics, 74, 901-910.

Volpe, J.J. (1995). Intracranial hemorrhage: germinal matrixintraventricular hemorrhage of the premature infant. In: J.J. Volpe (Ed.). Neurology of the newborn. 3rd ed (pp. 422-425). Philadelphia: WB Saunders Company.

Wildschut, H.I.J., Van Roosmalen, J., Van Leeuwen, E., \& Keirse, M.J.N.C. (1995). Planned abdominal compared with planned vaginal birth in triplet pregnancies. British Journal of Obstetrics and Gynaecology, 102, 292-296. 